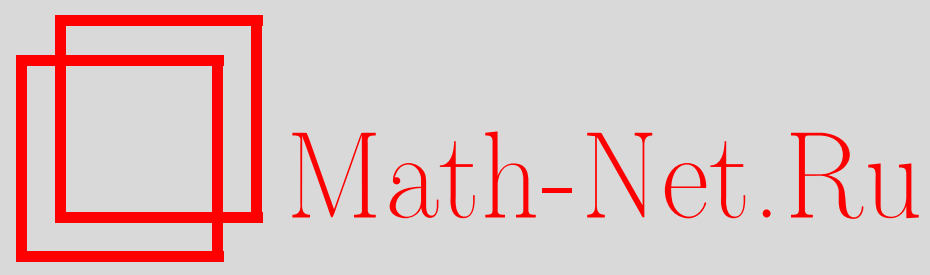

Б. Н. Хабибуллин, Полнота систем целых функций в пространствах голоморфных функций, Матем. заметки, 1999, том 66, выпуск 4, 603-616

DOI: https://doi.org/10.4213/mzm2815

Использование Общероссийского математического портала Math-Net.Ru подразумевает, что вы прочитали и согласны с пользовательским соглашением http://www.mathnet.ru/rus/agreement

Параметры загрузки:

IP : 34.227 .88 .159

26 апреля 2023 г., 13:09:39

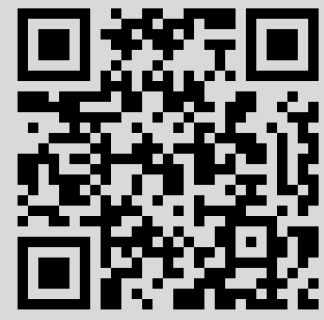




\section{ПОЛНОТА СИСТЕМ ЦЕЛЫХ ФУНКЦИЙ \\ В ПРОСТРАНСТВАХ ГОЛОМОРФНЫХ ФУНКЦИЙ}

\section{Б. Н. Хабибуллин}

Пусть $f$ - целая функция в $\mathbb{C}^{n}, n \geqslant 1, \Lambda \subset \mathbb{C}^{n}, E \subset \mathbb{C}^{n}$. В терминах взаимного индикатора функции $f$ и множества $E$, широкого класса плотностей распределения множества $\Lambda$ дается шкала достаточных условий полноты системы функций $\{f(\lambda \times z)$ : $\lambda \in \Lambda\}, z \in E$, где $\lambda \times z=\left(\lambda_{1} z_{1}, \lambda_{2} z_{2}, \ldots, \lambda_{n} z_{n}\right)$, в пространстве $H(E)$ голоморфных на $E$ функций в топологии равномерной сходимости на компактах. Эти условия новые уже при $n=1$, даже когда $E$ - круг.

Библиография: 19 названий.

Введение. Пусть $E \subset \mathbb{C}^{n}$. Через $H(E)$ обозначаем пространство голоморфных на $E$ функций, т.е. функций, голоморфных в открытой окрестности $E$. Говорим, что система функций $\mathscr{F}$ полна на (или в) множестве $E \in \mathbb{C}^{n}$, если для любой функции $g \in H(E)$ существует последовательность конечных линейных комбинаций с комплексными коэффициентами функций из системы $\mathscr{F}$, сходящаяся к $g$ в топологии равномерной сходимости на компактах из $E$.

Пусть $f$ - целая функция в $\mathbb{C}^{n}, n \geqslant 1$.

Первые результаты при $n=1$ о полноте в круге заданного радиуса с центром в нуле системы функций $\left\{f\left(\lambda_{k} z\right)\right\}$, где $\Lambda=\left\{\lambda_{k}\right\}$ - последовательность комплексных чисел, стремящаяся к бесконечности, были получены, по-видимому, А. О. Гельфондом и А.И. Маркушевичем [1], [2] (здесь мы опускаем обсуждение обширного материала для случая явно заданных функций $f$ типа $f(z)=e^{z}$ ). Дальнейшие исследования при $n=1$, когда полнота рассматривалась в круге или на всей комплексной плоскости, проводились И. И. Ибрагимовым [3], [4, гл. IV , § 3, п. 2], Б. Я. Левиным [5, с. 283], А. Ф. Леонтьевым [6], [7, гл. II, $\S 1]$. Полнота в областях на плоскости, отличных от круга и плоскости, изучалась И. Ф. Лохиньгм [8], [9] и И.И. Ибрагимовьм [4, гл. IV, § 3, п. 5].

В работах автора установлена

Теорема А. Пусть $G-$ выпуклая область в $\mathbb{C}$ с опорной функиией $h(-\theta)$,

$$
s_{h}(\theta)=h^{\prime}(\theta-0)+\int_{0}^{\theta} h(\varphi) d \varphi, \quad 0 \leqslant \theta \leqslant 2 \pi
$$

Работа выполнена при поддержке Российского фонда фундаментальных исследований, грант № 01-960-011746, и Конкурсного центра фундаментального естествознания при Санкт-Петербургском государственном университете, грант № 01-960-000936. 
$\left\{\lambda_{q}\right\}$ - последовательность попарно различных комплексных чисел, $k(\theta)-2 \pi$-периодическая $\gamma$-тригонометрически выпуклая неотрицательная функиия,

$$
\Lambda(t ; k) \stackrel{\text { def }}{=} \sum_{\left|\lambda_{q}\right|<t} k\left(\arg \lambda_{q}\right) .
$$

Если выполнено неравенство

1) при $\gamma<1$

$$
\varlimsup_{r \rightarrow+\infty} \frac{1}{2 r} \int_{1}^{r}\left(\left(\frac{r}{t}\right)^{\gamma}+\left(\frac{t}{r}\right)^{\gamma}\right) \frac{\Lambda(t ; k)}{t} d t \geqslant \frac{1}{1-\gamma^{2}} \frac{1}{2 \pi} \int_{0}^{2 \pi} k(\theta) d s_{h}(\theta),
$$

или

2) $n p u \gamma=1$

$$
\varlimsup_{a \rightarrow+\infty} \frac{1}{\ln a} \varlimsup_{r \rightarrow+\infty} \int_{r}^{a r} \frac{\Lambda(t ; k)}{t^{2}} d t \geqslant \frac{1}{2 \pi} \int_{0}^{2 \pi} k(\theta) d s_{h}(\theta),
$$

илu

3) прu $\gamma>1 u\|k\|=\max _{0 \leqslant \theta \leqslant 2 \pi} k(\theta)$

$$
\begin{gathered}
\varlimsup_{r \rightarrow+\infty} \frac{1}{2 r}\left(2\|k\| \int_{1}^{r} \frac{\Lambda(t ; 1)}{t} d t-\int_{1}^{r}\left(\frac{t}{r}\right)^{\gamma} \frac{\Lambda(t ; k)}{t} d t+\int_{r}^{+\infty}\left(\frac{r}{t}\right)^{\gamma} \frac{\Lambda(t ; k)}{t} d t\right) \\
\geqslant\|k\| \frac{1}{2 \pi} \int_{0}^{2 \pi} h(\theta) d \theta+\frac{1}{\gamma^{2}-1} \frac{1}{2 \pi} \int_{0}^{2 \pi} k(\theta) d s_{h}(\theta),
\end{gathered}
$$

то система $\left\{\exp \left(\lambda_{q} z\right)\right\}$ полна в области $G$.

Первое и второе условие полноты были получены в [10, теорема 4.1]. Впрочем, в силу известной связи между полнотой систем экспонент в области и теоремами единственности [2] второе условие полноты в теореме А было известно и несколько раньше как теорема единственности из совместной работы А. Ф. Гришина и М. Л. Содина [11]. Последнее условие также в виде теоремы единственности впервые установлено в несколько иной форме в $[12$, теорема единственности, 3$)]$, которая интегрированием по частям легко сводится к виду $(0.5)$.

Основная цель настоящей работы - расширение теоремы А до условий полноты системы функций вида $\{f(\lambda \times z): \lambda \in \Lambda\}$ в области Рунге $G \subset \mathbb{C}^{n}$ или на полиномиально выпуклом компакте $K \subset \mathbb{C}^{n}$, где $f$ - целая функция в $\mathbb{C}^{n}, n \geqslant 1$, конечного типа при порядке $\rho, \Lambda \subset \mathbb{C}^{n}$,

$$
\lambda \times z \stackrel{\text { def }}{=}\left(\lambda_{1} z_{1}, \lambda_{2} z_{2}, \ldots, \lambda_{n} z_{n}\right) .
$$

При этом в п. 2 уже в случае $n=1$ теорема А дополняется новыми условиями полноты системы функций вида $\left\{f\left(\lambda_{q} z\right): \Lambda=\left\{\lambda_{q}\right\} \subset \mathbb{C}, q \in \mathbb{N}\right\}$ в области $G$.

При $n>1$ основная теорема о полноте имеет смысл только в случае, когда множество $\Lambda$ имеет ненулевую меру Лебега-Хаусдорфа порядка $2 n-2$ и существенно отличается от ситуации дискретного вешественного множества $\Lambda$ (см. обзор [13, п. 5.3]).

В последнем пункте статьи схематически рассмотрено возможное обобщение теоремы о полноте на вопросы полноты системы функций вида $\left\{f(z, \lambda): \lambda \in \Lambda \subset \mathbb{C}^{n}\right\}$. 
1. Взаимный индикатор плюрисубгармонической или целой функции и множества. Пусть $u$ - плюрисубгармоническая функция в $\mathbb{C}^{n}, n \geqslant 1$ (субгармоническую в $\mathbb{C}$ функцию также называем плюрисубгармонической), $E$ - множество в $\mathbb{C}^{n}$. Положим

$$
B(\lambda ; u, E) \stackrel{\text { def }}{=} \sup _{z \in E} u(\lambda \times z), \quad B^{*}(\lambda ; u, E) \stackrel{\text { def }}{=} \varlimsup_{\lambda^{\prime} \rightarrow \lambda} B\left(\lambda^{\prime} ; u, E\right), \quad \lambda \in \mathbb{C}^{n},
$$

- полунепрерывная сверху регуляризация функции $B(\lambda ; \cdot, \cdot)$. Из простых свойств плюрисубгармонических функций следует

ПРЕДЛОЖЕНИЕ 1.1. В введенныХ обозначениях

1) функиия $B^{*}(\lambda ; u, E)$ плюрисубгармоническая во внутренности того множества, где она локально ограничена сверху;

2) если $E$ - ограниченное множество, то $B^{*}(\lambda ; u, E)$ - плюрисубгармоническая фукиия в $\mathbb{C}^{n}$

3) если $Е$ - ограниченное множсество и и - функиия конечного типа при порядке $\rho$, то такова же и функция $B^{*}(\lambda ; u, E)$.

ОПРЕДЕЛЕНИЕ 1.1. Пусть число $\rho>0$ и функция

$$
H_{\rho}(\lambda ; u, E) \stackrel{\text { def }}{=} \varlimsup_{t \rightarrow+\infty} \frac{B^{*}(t \lambda ; u, E)}{t^{\rho}}, \quad \lambda \in \mathbb{C}^{n} .
$$

Взаимным индикатором функиии и и множества $E$ при порядке $\rho$ назьваем ее полунепрерьвную сверху регуляризацию

$$
H_{\rho}^{*}(\lambda ; u, E) \stackrel{\text { def }}{=} \varlimsup_{\lambda^{\prime} \rightarrow \lambda} H_{\rho}\left(\lambda^{\prime} ; f, G\right), \quad \lambda \in \mathbb{C}^{n} .
$$

Если функция $u$ конечного типа при порядке $\rho, E$ - ограниченное множество, то их взаимньй индикатор есть регуляризованньй радиальный индикатор функции $B^{*}(\lambda ; u, E)$.

Если $E=\{(1,1, \ldots, 1)\}$ - одноточечное множество, то взаимньй индикатор функции $u$ и множества $E$ при порядке $\rho$ есть обычньй регуляризованньй радиальный индикатор функции $u$ при порядке $\rho$.

Если $\rho=1, f(z)=\exp \left(z_{1}+z_{2}+\cdots+z_{n}\right), z \in \mathbb{C}^{n}$, a $E$ - вьпуклая область или вьпуклый компакт в $\mathbb{C}^{n}$, то взаимный индикатор этой функции и $E$ при порядке 1 совпадает с опорной функцией множества $E$.

Отметим свойства взаимного индикатора.

ПРЕДЛОЖЕНИЕ 1.2. Взаимный индикатор $H_{\rho}^{*}(\lambda ; u, E)$

1) плюрисубгармоничен во внутренности того множества, где он локально ограничен сверху;

2) плюрисубгармоническая функиия конечного типа при порядке $\rho$, если и конечного типа при порядке $\rho$ и Е ограничено;

3) положительно однороден степени $\rho$, т.е. $H_{\rho}^{*}(t \lambda ; \cdot, \cdot)=t^{\rho} H_{\rho}^{*}(\lambda ; \cdot, \cdot), t>0$. 
Если $H$ - непрерывная полохстельно однородная степени $\rho$ функиия на $\mathbb{C}^{n}$ такая, что $H_{\rho}^{*} \leqslant H$ на $\mathbb{C}^{n}$, то для любого $\varepsilon>0$ найдется постоянная $C_{\varepsilon}$ такая, что $B^{*}(\lambda ; u, E) \leqslant H(\lambda)+\varepsilon|\lambda|^{\rho}+C_{\varepsilon}$. При $n=1$ в әтом неравенстве функиию $H$ можнно заменить на взаимный индикатор, поскольку он непрерывен.

Перечисленные свойства следуют из определения взаимного индикатора и элементарных свойств плюрисубгармонических функций и радиального индикатора (см. [14]).

Если плюрисубгармоническая функция $u=\log |f|$, где $f$ - целая функция, то всюду ниже в функциях $B(\lambda ; u, \cdot), B^{*}(\lambda ; u, \cdot), H_{\rho}(\lambda ; u, \cdot)$ и $H_{\rho}^{*}(\lambda ; u, \cdot)$ допускается замена $u$ на $f$. При этом функцию $H_{\rho}^{*}(\lambda ; f, E)$ называем взаимным индикатором иелой функиии $f$ и множества $E$ при порядке $\rho$.

2. Теорема о полноте для комплексной плоскости. Пусть $h-2 \pi$-периодическая $\rho$-тригонометрически выпуклая функция, $\rho \geqslant 0$ [5]. С функцией $h$ ассоциируется неотрицательная мера $d s_{h}^{(\rho)}(\theta)$ на окружности:

$$
s_{h}^{(\rho)}(\theta)=h^{\prime}(\theta-0)+\rho^{2} \int_{0}^{\theta} h(\varphi) d \varphi, \quad 0 \leqslant \theta \leqslant 2 \pi .
$$

Для непрерьвной функции $k(\theta)$ положим (ср. с правыми частями в $(0.3)-(0.5))$

$$
S_{\rho}(k, h) \stackrel{\text { def }}{=} \frac{1}{2 \pi} \int_{0}^{2 \pi} k(\theta) d s_{h}^{(\rho)}(\theta) .
$$

ОПРЕДЕЛЕНИЕ 2.1. Верхнюю $(k, \rho)$-плотность $\bar{D}_{k}(\Lambda ; \rho)$ последовательности $\Lambda=$ $\left\{\lambda_{q}\right\}, q \in \mathbb{N}, \lambda_{q}=t_{q} e^{i \theta_{q}} \rightarrow \infty$, для $2 \pi$-периодической $\gamma$-тригонометрически вьпуклой неотрицательной функции $k$, при которой функция $\Lambda(t ; k)$ задана соотношением $(0.2)$ и $\|k\| \stackrel{\text { def }}{=} \max \{k(\theta): 0 \leqslant \theta \leqslant 2 \pi\}$, определяем следуюшим образом:

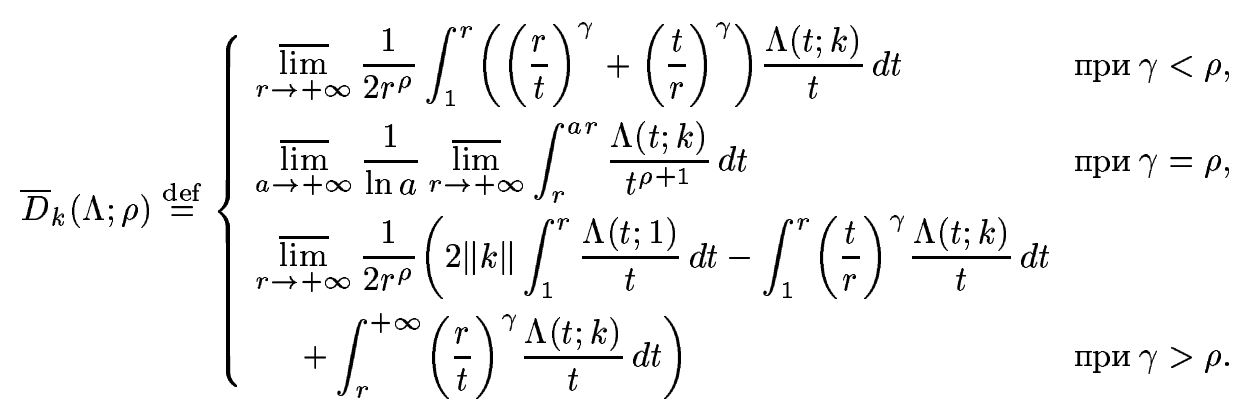

Легко видеть, что в определении 2.1 при $\rho=1$ получаются в точности левые части соотношений (0.3)-(0.5).

Определению 2.1 можно придать и иную форму, не использующую функцию $\Lambda(t ; k)$, а именно: 
ОПРЕДЕЛЕниЕ $2.1^{\prime}$. В обозначениях и условиях определения 2.1 верхнюю $(k, \rho)$-плотность $\bar{D}_{k}(\Lambda ; \rho)$ можно определить так:

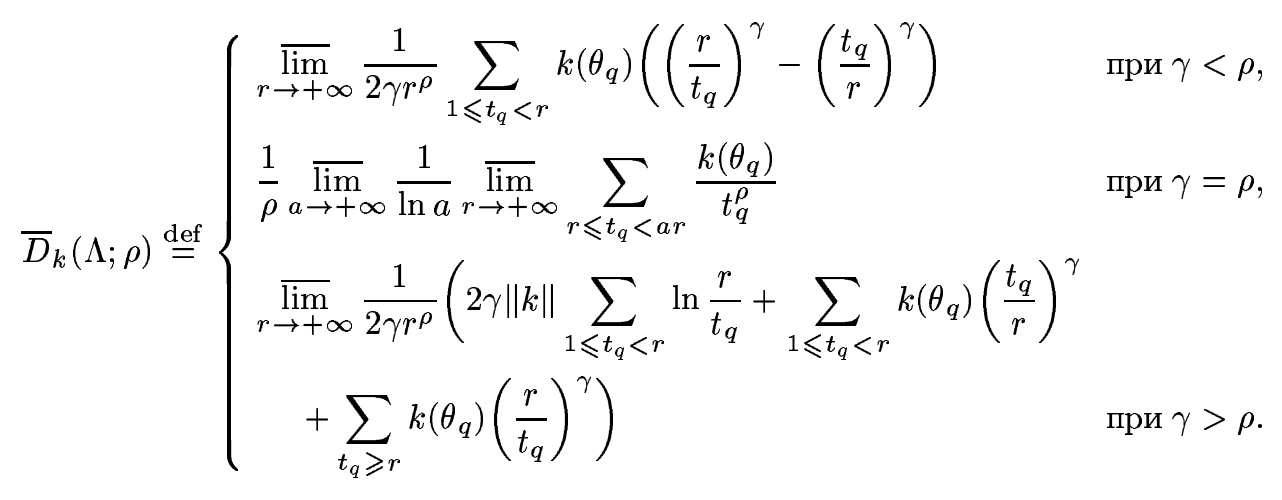

Переход от определения $2.1^{\prime}$ к определению 2.1 нетрудно получить интегрированием по частям.

ТЕОРема 2.1 (о полноте). Пусть $f$ - целая функиия на комплексной плоскости конечного типа при порядке $\rho>0$ и все ее тейлоровские коэффичиенты $a_{m}$ отличны от $0, E$ - односвязная область (соответственно компакт со связным дополнением) в $\mathbb{C}, \Lambda=\left\{\lambda_{q}\right\}$ - последовательность попарно различных комплексных чисел, $\lambda_{q} \rightarrow \infty, k-2 \pi$-периодическая $\gamma$-тригонометрически выпуклая неотрицательная функиия, $h$ - сужение на единичную окружность взаимного индикатора функиии $f$ и множества $E$ при порядке $\rho$. Если выполнено неравенство

1) $n p u \gamma<\rho$

$$
D_{k}(\Lambda ; \rho)>\frac{1}{\rho^{2}-\gamma^{2}} S_{\rho}(k, h),
$$

или

2) $n p u \gamma=\rho$

$$
D_{k}(\Lambda ; \rho)>\frac{1}{\rho} S_{\rho}(k, h)
$$

или

3) прu $\gamma>\rho$

$$
D_{k}(\Lambda ; \rho)>\frac{\|k\|}{\rho^{2}} S_{\rho}(1, h)+\frac{1}{\gamma^{2}-\rho^{2}} S_{\rho}(k, h),
$$

то система функиий $\left\{f\left(\lambda_{q} z\right)\right\}$ полна в области $E$ (соответственно в пространстве $A(E)$ голоморфных во внутренности компакта $E$ и непрерывных на компакте функиий в sup-норме).

ДокАЗАТЕЛЬСТво. Пусть $\nu$ - линейный непрерьвньй функционал на пространстве $H(E)$ (соответственно $A(E)$, если $E$ - компакт), аннулирующий систему $\left\{f\left(\lambda_{q} z\right)\right\}$. Он реализуется как комплекснозначная конечная борелевская $\sigma$-аддитивная мера с носителем, компактно вложенным в E. Рассмотрим функцию

$$
F(\lambda)=\int_{\operatorname{supp} \nu} f(\lambda z) d \nu(z), \quad \lambda \in \mathbb{C} .
$$


Функция $F$ целая и $F\left(\lambda_{q}\right)=0, q \in \mathbb{N}$. В обозначениях п. 1 имеем

$$
|F(\lambda)| \leqslant \int_{\operatorname{supp} \nu}|f(\lambda z)| d|\nu|(z) \leqslant|\nu|(\mathbb{C}) \exp B^{*}(\lambda ; F, \operatorname{supp} \nu) .
$$

Отсюда по предложению 1.1 следует, что $F$ - целая функция конечного типа при порядке $\rho$. В силу непрерьвности $2 \pi$-периодической $\rho$-тригонометрически вьпуклой функции $h$ из предложений 1.1 и 1.2 следует, что для любого $\varepsilon>0$ найдется постоянная $C_{\varepsilon}$ такая, что

$$
B^{*}\left(t e^{i \theta} ; F, \operatorname{supp} \nu\right) \leqslant(h(\theta)+\varepsilon) t^{\rho}+C_{\varepsilon}, \quad t \geqslant 0, \quad 0 \leqslant \theta \leqslant 2 \pi .
$$

Согласно (2.7) это означает, что индикатор роста $h_{F}$ целой функции $F$ при порядке $\rho$ не превышает $h$, т.е. $S_{\rho}\left(k, h_{F}\right) \leqslant S_{\rho}(k, h)$ ввиду неотрицательности функции $k$. Тем самым, выполнение условий теоремы 2.1 влечет выполнение одного из неравенств $(2.3)-(2.5)$ с заменой $h$ на $h_{F}$. По теореме единственности, доказанной в конце работы [12] (там используется определение в форме $2.1^{\prime}$ ), это означает, что $F(\lambda) \equiv 0$. Раскладьвая целую функцию $f$ по степеням, из (2.6) получаем

$$
\sum_{m=0}^{\infty} a_{m} \lambda^{m} \int_{\operatorname{supp} \nu} z^{m} d \nu(z)=0, \quad \lambda \in \mathbb{C}
$$

Так как $a_{m} \neq 0$ при всех $m$, мера $\nu$ аннулирует все степени $z^{m}$, которые полны в $H(E)$, когда $E$ - односвязная область, и полны в $A(E)$ по теореме Мергеляна, если $E$ - компакт. Значит $\nu=0$ и по известному следствию теоремы Хана-Банаха система функций $\left\{f\left(\lambda_{q} z\right)\right\}$ полна.

Теорема доказана.

3. Формулировка теоремы о полноте для многих переменных и теорема единственности. Пусть $S^{m-1}$ - единичная сфера в $\mathbb{R}^{m}$ с центром в нуле, $D^{\prime}\left(S^{m-1}\right)-$ пространство обобщенных функций на $S^{m-1}$.

ОПРЕДЕЛЕниЕ 3.1 [16]. Полунепрерьвная сверху интегрируемая на $S^{m-1}$ функция $h: S^{m-1} \rightarrow[-\infty,+\infty)$ называется субсферической функиией порядка $\rho$, если $\mathscr{L}_{\rho} h \geqslant 0$, где оператор $\mathscr{L}_{\rho}$ определяется как $\mathscr{L}_{\rho}=\Delta_{\theta}+\rho(\rho+m-2), \Delta_{\theta}-$ сферическая часть оператора Лапласа [15, с. 115], а неравенство вьполнено в $D^{\prime}\left(S^{m-1}\right)$. При этом меру $s_{h}^{(\rho)}=\mathscr{L}_{\rho} h$ на $S^{m-1}$ называем ассоциированной с $h$ мерой (аналог $(0.1)$ и (2.1)).

Класс всех (соответственно неотрищательных) субсферических функций порядка $\rho$ обозначим через $\mathrm{SS}_{\rho}$ (соответственно через $\mathrm{SS}_{\rho}^{+}$).

Другие эквивалентные определения субсферических функций и их свойства имеются в [10], [16]. Из них следует, что если $\gamma \leqslant \rho$, то $_{\gamma}^{+} \subset \mathrm{SS}_{\rho}^{+}$. Поэтому далее считаем, что каждая субсферическая функция задается вместе с ее порядком. Субсферические функции порядка 0 - это постоянные.

Функция $h$ - субсферическая функция порядка $\rho>0$ тогда и только тогда, когда функция $H(x)=h(x /|x|)|x|^{\rho}, H(0)=0, \rho>0$, где $|x|-$ евклидова норма элемента $x \in \mathbb{R}^{m}$, является субгармонической функцией в $\mathbb{R}^{m}[10]$. 
При $m=2$ класс субсферических функций порядка $\rho$ совпадает с классом $2 \pi$-периодических $\rho$-тригонометрически вьпуклых функций. Примерами субсферических функций порядка $\rho$ служат сужения на единичную сферу индикаторов (плюри)субгармонических и целых функций конечного типа при порядке $\rho[16],[10]$, и при $\rho=1$ опорных функций вьпуклых ограниченных множеств из $\mathbb{R}^{m}$. По предложению 1.2 сужения на единичную сферу в $\mathbb{C}^{n}$, отождествленном с $\mathbb{R}^{2 n}$, взаимного индикатора $H_{\rho}^{*}(\lambda ; u, E)-$ функции из $\mathrm{SS}_{\rho}$, если $u$ конечного типа при порядке $\rho$ и $E$ ограничено.

Для полунепрерьвной сверху функции $k$ на $S^{m-1}$ при $h \in \mathrm{SS}_{\rho}$ полагаем

$$
S_{\rho}(k, h) \stackrel{\text { def }}{=} \frac{1}{2 \pi} \int_{S^{m-1}} k d s_{h}^{(\rho)} .
$$

Это многомерньй аналог (2.2) и правой части (0.4).

Через $\sigma_{p}$ обозначаем меру Лебега-Хаусдорфа порядка $p$ в $\mathbb{R}^{m}$, т.е. для множества $E \subset \mathbb{R}^{m}$

$$
\sigma_{p}(E) \stackrel{\text { def }}{=} \lim _{\varepsilon \rightarrow 0} \sigma_{p}^{\varepsilon}(E), \quad \sigma_{p}^{\varepsilon}(E) \stackrel{\text { def }}{=} \inf \omega_{p} \sum_{j} r_{j}^{p},
$$

где инфимум берется по всем покрытиям множества $E$ не более чем счетными множествами шаров из $\mathbb{R}^{m}$ радиусов $r_{j}<\varepsilon, \omega_{p}$ - объем единичного шара в $\mathbb{R}^{p}[17$, п. 6$]$.

Пусть $k$ - неотрицательная субсферическая функция порядка $\gamma$ на единичной сфеpe $S^{2 n-1}$ из $\mathbb{C}^{n}$, отождествленного с $\mathbb{R}^{2 n}$, т.е. $k$ - функция на $S^{2 n-1} \subset \mathbb{C}^{n}$,

$$
\|k\| \stackrel{\text { def }}{=} \sup \left\{k(\zeta): \zeta \in S^{2 n-1}\right\} .
$$

Для $\Lambda \subset \mathbb{C}^{n}, n>1$, аналогично (0.2) полагаем

$$
\Lambda(t ; k) \stackrel{\text { def }}{=} \int_{\Lambda \cap t \mathbf{B}} k\left(\frac{z}{|z|}\right) d \sigma_{2 n-2}(z),
$$

где $\mathbf{B}$ - единичньй открытьй шар в $\mathbb{C}^{n}$.

ОПРЕДЕЛЕНИЕ 3.2. Верхнюю $(k, \rho)$-плотность $\bar{D}_{k}(\Lambda ; \rho)$ множества $\Lambda \subset \mathbb{C}^{n}$ для функции $k \in \mathrm{SS}_{\gamma}^{+}$определим следующим образом:

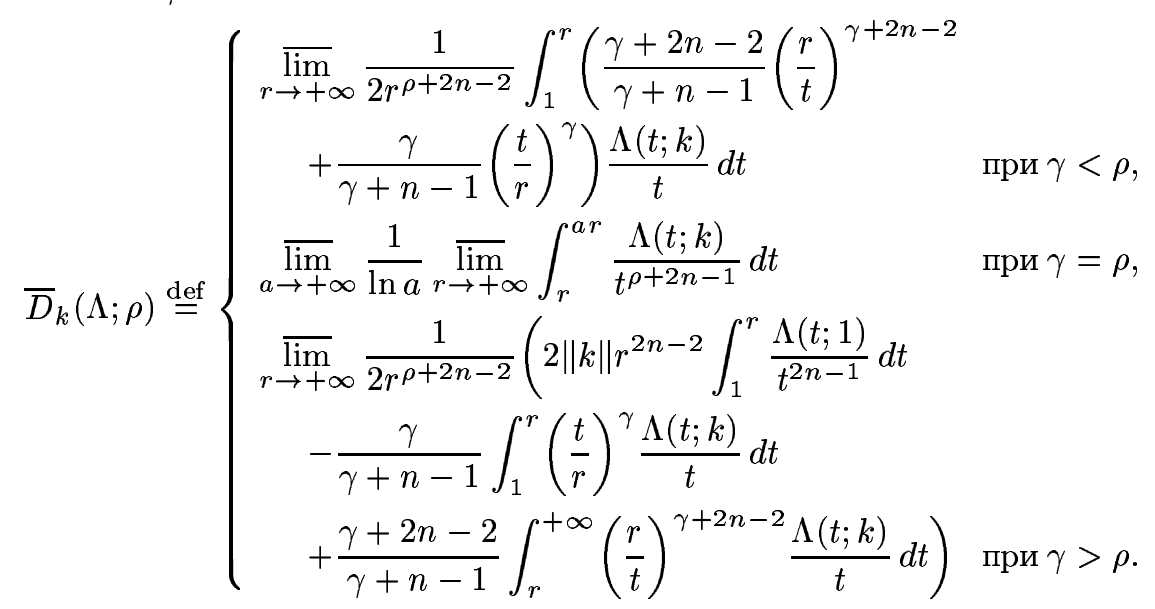

Легко видеть, что при $n=1$ верхняя $(k, \rho)$-плотность $\bar{D}_{k}(\Lambda ; \rho)$ в точности совпадает с определением 2.1. 
Теорема 3.1 (о полноте). Пусть $f$ - целая функиия в $\mathbb{C}^{n}, n>1$, конечного типа при порядке $\rho>0$ и все частные производные (по комплексным переменным) функиии $f$ в нуле отличны от $0, E$ - область Рунге или полиномиально выпукльй компакт в $\mathbb{C}^{n}, \Lambda \subset \mathbb{C}^{n}, h^{*}$ - сужение на единичную сферу $S^{2 n-1} \subset \mathbb{C}^{n}$ взаимного индикатора функции $f$ и множества $E$ при порядке $\rho, h$ - непрерывная субсферическая функиия порядка $\rho$ и $h^{*} \leqslant h$ на $S^{2 n-1}, k \in \mathrm{SS}_{\gamma}^{+}$. Если выполнено неравенство

1) $n p u \gamma<\rho$

$$
\bar{D}_{k}(\Lambda ; \rho)>\frac{1}{(\rho-\gamma)(\rho+\gamma+2 n-2)} S_{\rho}(k, h)
$$

или

2) $n p u \gamma=\rho$

$$
\bar{D}_{k}(\Lambda ; \rho)>\frac{1}{\rho+2 n-2} S_{\rho}(k, h)
$$

uлu

3) прu $\gamma>\rho$

$$
\bar{D}_{k}(\Lambda ; \rho)>\frac{\|k\|}{\rho(\rho+2 n-2)} S_{\rho}(1, h)+\frac{1}{(\gamma-\rho)(\gamma+\rho+2 n-2)} S_{\rho}(k, h),
$$

то система функиий $\{f(\lambda \times z): \lambda \in \Lambda\}$ полна на $E$.

При подстановке $n=1$ эта теорема совпадает с формулировкой теоремы 2.1 с несколько более мягкими условиями на топологию пространства функций на $E$, когда $E-$ компакт. Ограничение $h^{*} \leqslant h$ при $n=1$ несущественно, так как в этом случае функция $h^{*}$ всегда непрерьвна.

Для доказательства теоремы 3.1 нам потребуется

Теорема 3.2 (единственности). Пусть $F$ - иелая функиия в $\mathbb{C}^{n}, n>1$, конечного типа при порядке $\rho>0$ с радиальным регуляризованным индикатором (при порядке $\rho>0)$, не превышающим непрерывную функиию $h \in \mathrm{SS}_{\rho}$. Eсли $\Lambda$ - подмножество нулевого множества $\Lambda_{F}=\left\{z \in \mathbb{C}^{n}: F(z)=0\right\}$ функиии $F$ и для $k \in \mathrm{SS}_{\gamma}^{+}$выполнено одно из соотношений 1)-3) теоремы 3.1, то $F(z) \equiv 0$.

ДокАЗАТЕЛЬСТво (случай $\gamma \leqslant \rho$ ). Из условий на рост целой функции $F$ следует, что в силу непрерывности положительно однородной степени $\rho$ функции $h$ для любого $\varepsilon>0$ сушествует постоянная $C_{\varepsilon}$, для которой (см. [14, следствие 7.3]) $\ln |F(z)| \leqslant$ $h_{\varepsilon}(z /|z|)+C_{\varepsilon}, z \in \mathbb{C}^{n}$, где $h_{\varepsilon}=h+\varepsilon$. Легко показать, что при $\varepsilon \rightarrow 0$ ассоциированная c $h_{\varepsilon}$ мера слабо сходится к мере $s_{h}^{(\rho)}$. Поэтому строгие неравенства в 1)-3) справедливы и после замены $h$ на $h_{\varepsilon}$ для достаточно малых $\varepsilon$. Следовательно, не умаляя общности, можно считать в условиях теоремы вьполненным неравенство

$$
\ln |F(z)| \leqslant h\left(\frac{z}{|z|}\right)|z|^{\rho}+\text { const, } \quad z \in \mathbb{C}^{n} .
$$

Обозначим через $\mu_{F}$ распределение масс Рисса субгармонической функции $\ln |F|$, если $F$ - ненулевая функция, и введем функцию

$$
\mu(t ; k) \stackrel{\text { def }}{=} \int_{t \mathbf{B}} k\left(\frac{z}{|z|}\right) d \mu_{F}(z) .
$$


Из известной формулы Пуанкаре-Лелона следует (см. рассуждения в конще [18, § 3]), что ввиду совпадения меры Лебега-Хаусдорфа порядка $2 n-2$ на аналитическом множестве $\Lambda_{F}$ с мерой, задаваемой $(2 n-2)$-мерной площадью на $\Lambda_{F}$ в евклидовой метрике, справедливо соотношение

$$
\Lambda(t ; k) \leqslant \frac{\theta_{2 n}}{2 \pi} \mu(t ; k)
$$

где $\theta_{2}=2 \pi$, а при $m \geqslant 3 \theta_{m}=(m-2) s_{m}, s_{m}$ - плошадь сферы $S^{m-1} \subset \mathbb{R}^{m}$.

В введенных обозначениях соотношения 1) или 2) теоремы 3.1 при условии (3.2) в точности совпадают с соответствующими условиями теоремы единственности для субгармонической функции $u=\ln |F|$ из [10] после определенной перенормировки. Применяя эту теорему, из ее заключения имеем $u \equiv-\infty$, что и завершает доказательство теоремы 3.2 в случае $\gamma \leqslant \rho$.

Доказательство теоремы 3.2 при $\gamma>\rho$ не следует из теоремы единственности из [10] для субгармонических функций и требует некоторой подготовки. Оно приводится в следующем пункте.

\section{4. Специальные функции Йенсена и доказательство теоремы 3.2 при $\gamma>\rho$.}

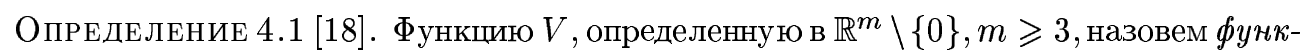
иией Йенсена (относительно точки 0), если вьполнены следующие условия:

1) $V$ субгармонична и неотрицательна в $\mathbb{R}^{m} \backslash\{0\}$;

2) $V(y) \leqslant|y|^{-m+2}+O(1),|y| \rightarrow 0(|y|-$ евклидова норма $y)$;

3) для любого $\varepsilon>0$ существует компакт $K_{\varepsilon}$ такой, что $V(y) \leqslant \varepsilon$ при $y \in \mathbb{R}^{m} \backslash K_{\varepsilon}$. Класс всех функций Йенсена обозначим через $\mathscr{J}$.

ПРЕДЛОЖЕНИЕ 4.1. Если $V$ - функиия Йенсена на $\mathbb{C}^{n}$, отождествленном $c \mathbb{R}^{2 n}$, $n>1$, то при любом $r>0$ функиия $r^{2-2 n} V(\zeta / r)$ - также функиия Йенсена по переменной $\zeta \in \mathbb{C}^{n}$.

Сохранение свойств из определения 4.1, характеризующих функции Йенсена, в предложении 4.1 очевидны.

Для субгармонической в $\mathbb{R}^{m}$ функции $u$ через $\mu_{u}$ обозначим распределение масс Рисса функции $u$, т.е. $\mu_{u}=\theta_{m}^{-1} \Delta u$ в смысле теории распределений.

Для целой функции $f$ в $\mathbb{C}^{n}$ положим $\Lambda_{f}=\left\{z \in \mathbb{C}^{n}: f(z)=0\right\}$ - нулевое множество функции $f$.

Важность понятия функций Йенсена связана со следующей оценкой нулевых множеств, которая в [18] доказана для голоморфных в области функций.

ТЕОремА Б [18]. Пусть $n>1, u$-субгармоническая, а $f$-иелая в $\mathbb{C}^{n}$ функиии, $f(z) \not \equiv 0$, функция $и$ ограничена в единичном шаре $\mathbf{B} \subset \mathbb{C}^{n}$. Ecли $\log |f| \leqslant u$ на $\mathbb{C}^{n}$, то найдется постоянная $C$ такая, что

$$
\int_{\Lambda_{f} \backslash \mathbf{B}} V d \sigma_{2 n-2} \leqslant \frac{2 \pi^{n-1}}{(n-2) !} \int_{\mathbb{C}^{n}} V d \mu_{u}+C \quad \text { для всех } \quad V \in \mathscr{J} .
$$


Введем в рассмотрение специальные функции Йенсена на $\mathbb{C}^{n}$. Пусть по определению $b^{+}=\max \{0, b\}, b \in \mathbb{R}$. Пусть $k-$ субсферическая функция на единичной сфере из $\mathbb{C}^{n}$ и $k \in \mathrm{SS}_{\gamma}^{+}$. Положим

$$
V_{k}(\zeta)= \begin{cases}\frac{n-1}{\gamma+n-1} \frac{k(\zeta /|\zeta|)}{\|k\|}|\zeta|^{-(\gamma+2 n-2)}, & \text { если }|\zeta| \geqslant 1, \\ \frac{n-1}{\gamma+n-1} \frac{k(\zeta /|\zeta|)}{\|k\|}|\zeta|^{\gamma}+\left(|\zeta|^{2-2 n}-1\right)^{+}, & \text {если } 0<|\zeta|<1 .\end{cases}
$$

ПРЕДЛОЖЕНИЕ 4.2. $V_{k}(\zeta)$ - функиия Йенсена по переменной $\zeta \in \mathbb{C}^{n}$.

ДокАЗАТЕЛЬСТВо. Неотрицательность этой функций и вьполнение условий 2) и 3) из определения 4.1 очевидны по построению этой функции. В доказательстве нуждается только ее субгармоничность при $\zeta \neq 0$.

Рассмотрим функцию

$$
W(\zeta)=W(t \theta)=k(\theta) t^{\gamma}, \quad t=|\zeta|, \quad \theta=\frac{\zeta}{|\zeta|} \in S^{2 n-1}
$$

Эта функция субгармоническая в $\mathbb{C}^{n}$ по одному из эквивалентньх определений субсферических функций порядка $\gamma[10$, предложение 1.3$]$. Тогда функция

$$
W^{*}(t \theta)=\frac{1}{t^{2 n-2}} W\left(\frac{\theta}{t}\right)=k(\theta) t^{-(\gamma+2 n-2)}, \quad t>0,
$$

субгармоническая в $\mathbb{C}^{n} \backslash\{0\}$ как преобразование Кельвина относительно единичной сферы субгармонической функции $W$ [19, с. 205].

Следовательно, функция $V_{k}$ субгармоническая всюду за исключением, быть может, нуля и единичной сферы, так как строится из функций $W$ при $|\zeta|>1$ и $W^{*}$ при $0<|\zeta| \leqslant 1$, а также гармонической при $0<|\zeta|<1$ функции $|\zeta|^{2-2 n}-1$.

При проверке субгармоничности на единичной сфере предположим сначала, что $k-$ функция класса $C^{2}$ на единичной сфере.

Нам потребуется

Лемма 4.1. Пусть $и$ - субгармоническая функиия в допустимых в смысле [19, 1.4.4] областях $D_{1}$ и $D_{2}$ из $\mathbb{R}^{m}, S$ - общая часть их границы и и непрерывно продолюсена на $D=D_{1} \cup S \cup D_{2}, \partial / \partial n_{i}$ - оператор дифференцирования по внешней нормали к области $D_{i}, i=1,2$. Если $u \in C^{2}$ в $\bar{D}_{i}, i=1,2$, и выполнено неравенство

$$
\frac{\partial u}{\partial n_{1}}+\frac{\partial u}{\partial n_{2}} \leqslant 0 \quad \text { на } S,
$$

то и-субгармоническая в $D$ функиия.

ДокАЗАТЕЛЬСтво. Пусть $\psi$ - бесконечно дифференцируемая финитная неотрицательная функция в $D$. По формуле Грина $[19,1.4 .4]$ имеем

$$
\int_{D_{i}}(u \Delta \psi-\psi \Delta u)=\int_{S}\left(u \frac{\partial \psi}{\partial n_{i}}-\psi \frac{\partial u}{\partial n_{i}}\right), \quad i=1,2 .
$$


Складьвая эти тождества, получаем

$$
\int_{D} u \Delta \psi=\int_{D_{1} \cup D_{2}} u \Delta \psi=\int_{D_{1} \cup D_{2}} \psi \Delta u-\int_{S} \psi\left(\frac{\partial u}{\partial n_{1}}+\frac{\partial u}{\partial n_{2}}\right) \geqslant 0
$$

т.е. $\Delta u \geqslant 0$, значит $u$ - субгармоническая в $D$ функция. Лемма доказана.

Согласно лемме для доказательства субгамоничности $V_{k}$ на единичной сфере достаточно показать, что сумма производных по внешней нормали к единичной сфере относительно областей $D_{1}=\mathbf{B} \backslash\{0\}$ и $D_{2}=\mathbb{C}^{n} \backslash \mathbf{B}$ неположительна.

При $t=|\zeta| \geqslant 1$ и $\theta=\zeta /|\zeta| \in S^{2 n-1}$ имеем

$$
\frac{\partial V_{k}}{\partial n_{2}}(\theta)=-\left.\frac{n-1}{\gamma+n-1} \frac{k(\theta)}{\|k\|} \frac{\partial t^{-(\gamma+2 n-2)}}{\partial t}\right|_{t=1}=(\gamma+2 n-2) \frac{n-1}{\gamma+n-1} \frac{k(\theta)}{\|k\|}
$$

а при $t=|\zeta| \leqslant 1$

$$
\frac{\partial V_{k}}{\partial n_{1}}(\theta)=\left.\frac{n-1}{\gamma+n-1} \frac{k(\theta)}{\|k\|} \frac{\partial t^{\gamma}}{\partial t}\right|_{t=1}+\left.\frac{\partial\left(t^{2-2 n}-1\right)}{\partial t}\right|_{t=1}=\gamma \frac{n-1}{\gamma+n-1} \frac{k(\theta)}{\|k\|}+(2-2 n) .
$$

Складьвая полученные значения производных по внешней нормали, получаем $2(n-1) \times$ $k(\theta) /\|k\|+(2-2 n) \leqslant 0$. По лемме это означает субгармоничность $V_{k}$ всюду вне нуля.

От предположения, что $k$ - функция класса $C^{2}$ на единичной сфере, легко избавиться, аптроксимируя произвольную функцию $k \in \mathrm{SS}_{\gamma}^{+}$монотонно убывающей последовательностью субсферических функций класса $C^{2}$ (см. [10, предложение 1.7]).

ДокАЗАТЕЛЬСТво ТЕОРЕмы 3.2 (при $\gamma>\rho$ ). Допустим, что $F$ - ненулевая целая функция. Так как $F$ конечного типа при порядке $\rho$, то вьполнены соотношения

$$
\Lambda(t ; k)=O\left(t^{\rho+2 n-2}\right), \quad t \rightarrow+\infty, \quad \text { и } \Lambda(1 ; k)<+\infty, \quad k \in \mathrm{SS}_{\gamma}^{+} .
$$

В начале доказательства теоремы в п. 3 было показано, что можно считать выполненным условие $(4.2)$, где функция $H(z)=h(z /|z|)|z|^{\rho}$ субгармоническая. Тогда по теореме Б и предложениям 4.1 и 4.2 при $r>0$ вьполнено

$$
\int_{\Lambda_{F} \backslash \mathbf{B}} V_{k}\left(\frac{\zeta}{r}\right) d \sigma_{2 n-2}(\zeta) \leqslant \frac{2 \pi^{n-1}}{(n-2) !} \int_{\mathbb{C}^{n}} V_{k}\left(\frac{\zeta}{r}\right) d \mu_{H}(\zeta)+\text { const } \cdot r^{2 n-2}
$$

Как отмечено в $[10,(3.4)]$,

$$
d \mu_{H}=\frac{1}{\theta_{2 n}} d s_{h}^{(\rho)} \otimes t^{\rho+2 n-3} d t, \quad \text { где } \theta_{2 n}=\frac{4 \pi^{n}}{(n-2) !} .
$$

Отсюда и из явного вида функции $V_{k}$ непосредственные вычисления в сферических координатах дают, что правая часть соотношения (4.2) равна

$$
\begin{aligned}
I_{R}(r)= & 2(n-1)\left(\frac{1}{\rho(\rho+2 n-2)} S_{\rho}(1, h)\right. \\
& \left.+\frac{1}{\|k\|(\gamma-\rho)(\gamma+\rho+2 n-2)} S_{\rho}(k, h)\right) r^{\rho+2 n-2}+\mathrm{const} \cdot r^{2 n-2}
\end{aligned}
$$


При этом левая часть (4.2) по определению функций $V_{k}$ при $r \geqslant 1$ равна

$$
\begin{aligned}
I_{L}(r)= & \int_{\substack{1 \leqslant|\zeta|<r \\
\zeta \in \Lambda_{F} \backslash \mathbf{B}}}\left(\left(\frac{r}{|\zeta|}\right)^{2 n-2}-1\right) d \sigma_{2 n-2}(\zeta) \\
& +\frac{n-1}{\|k\|(\gamma+n-1)}\left(\int_{\substack{1 \leqslant|\zeta|<r \\
\zeta \in \Lambda_{F} \backslash \mathbf{B}}}\left(\frac{|\zeta|}{r}\right)^{\gamma} k\left(\frac{\zeta}{|\zeta|}\right) d \sigma_{2 n-2}(\zeta)\right. \\
& \left.+\int_{\substack{|\zeta| \geqslant r \\
\zeta \in \Lambda_{F} \backslash \mathbf{B}}}\left(\frac{r}{|\zeta|}\right)^{\gamma+2 n-2} k\left(\frac{\zeta}{|\zeta|}\right) d \sigma_{2 n-2}(\zeta)\right)
\end{aligned}
$$

Используя (3.1), при $t=|\zeta|$ левую часть можно переписать в виде

$$
\begin{aligned}
I_{L}(r)= & \int_{1}^{r}\left(\left(\frac{r}{t}\right)^{2 n-2}-1\right) d \Lambda_{F}(t ; 1) \\
& +\frac{n-1}{\|k\|(\gamma+n-1)}\left(\int_{1}^{r}\left(\frac{t}{r}\right)^{\gamma} d \Lambda_{F}(t ; k)+\int_{r}^{+\infty}\left(\frac{r}{t}\right)^{\gamma+2 n-2} d \Lambda_{F}(t ; k)\right) .
\end{aligned}
$$

Отсюда, интегрируя по частям и учитывая (4.1), при $r \geqslant 1$ получаем

$$
\begin{aligned}
I_{L}(r)= & 2 r^{2 n-2}(n-1) \int_{1}^{r} \frac{\Lambda_{F}(t ; 1)}{t^{2 n-1}} d t+\frac{n-1}{\|k\|(\gamma+n-1)}\left(-\gamma \int_{1}^{r}\left(\frac{t}{r}\right)^{\gamma} \frac{\Lambda_{F}(t ; k)}{t} d t\right. \\
& \left.+(\gamma+2 n-2) \int_{r}^{+\infty}\left(\frac{r}{t}\right)^{\gamma+2 n-2} \frac{\Lambda_{F}(t ; k)}{t} d t\right)+O\left(r^{2 n-2}\right) .
\end{aligned}
$$

Из последнего соотношения, тождества (4.3) и неравенства $I_{L}(r) \leqslant I_{R}(r)$, совпадающего с (4.2), учитьвая определение верхней $(k, \rho)$-плотности при $\gamma>\rho$, после деления обех частей неравенства $I_{L}(r) \leqslant I_{R}(r)$ на $2(n-1) r^{\rho+2 n-2} /\|k\|$, устремляя $r$ к $+\infty$, получаем

$$
\bar{D}_{k}\left(\Lambda_{F} ; \rho\right) \leqslant \frac{\|k\|}{\rho(\rho+2 n-2)} S_{\rho}(1, h)+\frac{1}{(\gamma-\rho)(\gamma+\rho+2 n-2)} S_{\rho}(k, h) .
$$

В силу включения $\Lambda \subset \Lambda_{F}$ в последнем неравенстве $\Lambda_{F}$ можно заменить на $\Lambda$. Это противоречит соотношению 3 ) теоремы 3.1. Следовательно, $F$ - нулевая функция, и теорема 3.2 доказана.

\section{5. Доказательство теоремы 3.1 (о полноте) и ее обобщение.}

ДОКАЗАТЕЛЬСТВО ТЕОРЕМЫ 3.1. Пусть $\nu$ - линейный непрерьвньй функционал на пространстве $H(E)$, аннулируюший систему $\{f(\lambda \times z): \lambda \in \Lambda\}$. Он реализуется как комплекснозначная конечная борелевская $\sigma$-аддитивная мера с носителем, компактно вложенным в $E$, и

$$
\int_{\operatorname{supp} \nu} f(\lambda \times z) d \nu(z)=0, \quad \lambda \in \Lambda \text {. }
$$

Рассмотрим функцию

$$
F(\lambda)=\int_{\operatorname{supp} \nu} f(\lambda \times z) d \nu(z), \quad \lambda \in \mathbb{C}^{n}
$$


Функция $F$ целая и $F(\lambda)=0, \lambda \in \Lambda$. В обозначениях п. 1 имеем

$$
|F(\lambda)| \leqslant \int_{\operatorname{supp} \nu}|f(\lambda \times z)| d|\nu|(z) \leqslant|\nu|\left(\mathbb{C}^{n}\right) \exp B^{*}(\lambda ; F, \operatorname{supp} \nu) .
$$

Отсюда по предложению 1.1 следует, что $F$ - целая функция конечного типа при порядке $\rho$. В силу непрерьвности субсферической функции $h$ из предложений 1.1 и 1.2 и из [14, следствие 7.3] следует, что для любого $\varepsilon>0$ найдется постоянная $C_{\varepsilon}$ такая, что

$$
B^{*}(z ; F, \operatorname{supp} \nu) \leqslant\left(h\left(\frac{z}{|z|}\right)+\varepsilon\right)|z|^{\rho}+C_{\varepsilon}, \quad z \in \mathbb{C}^{n} .
$$

Согласно (5.2) это означает, что радиальньй регуляризованньй индикатор функции $F$ (при порядке $\rho>0$ ) не превьшшает непрерывную функцию $h \in \mathrm{SS}_{\rho}$. По теореме единственности $3.2 F(\lambda) \equiv 0$. Раскладывая целую функцию $f$ по степеням, из (5.1) получаем

$$
\sum_{m} a_{m} \lambda^{m} \int_{\operatorname{supp} \nu} z^{m} d \nu(z)=0, \quad \lambda \in \mathbb{C}^{n},
$$

где $m$ пробегает множество всех мультииндексов $(\mathbb{N} \cup\{0\})^{n}$, а все коэффициенты разложения $a_{m} \neq 0$ как частные производные в нуле функции $f$. Следовательно, мера $\nu$ аннулирует все степени $z^{m}$. Многочлены полны в области Рунге по определению, а на полиномиально вьпуклом компакте по теореме Ока-Вейля. Значит, $\nu=0$ и по известному следствию из теоремы Хана-Банаха система функций $\{f(\lambda \times z): \lambda \in \Lambda\}$ полна на $E$.

Теорема доказана.

Следуя И. И. Ибрагимову [4, гл. IV,$\S 3$, п. 5], укажем возможное обобщение теоремы 3.2 .

Пусть система функций $\left\{a_{m}(z): m \in(\mathbb{N} \cup\{0\})^{n}\right\}$, голоморфных на множестве $E \subset \mathbb{C}^{p}$, полна на $E$.

Для мультииндексов $m$ полагаем

$$
|m| \stackrel{\text { def }}{=} m_{1}+m_{2}+\cdots+m_{n}, \quad m=\left(m_{1}, m_{2}, \ldots, m_{n}\right) \in(\mathbb{N} \cup\{0\})^{n} .
$$

Пусть $\rho>0$. Предположим, что

$$
|m|^{1 / \rho}\left(\sup _{z \in E}\left|a_{m}(z)\right|\right)^{1 /|m|}=O(1), \quad m \in(\mathbb{N} \cup\{0\})^{n} .
$$

Тогда [15, теорема 3.1.1] ряд

$$
f(z, \lambda)=\sum_{m} a_{m}(z) \lambda^{m}, \quad \lambda \in \mathbb{C}^{n},
$$

сходится равномерно по $z \in E$ и, в частности, $f(z, \lambda)$ - целая функция конечного типа при порядке $\rho$ в $\mathbb{C}^{n}$ по $\lambda$ при каждом $z \in E$. Более того, величина типа целых по $\lambda$ функций $f(z, \lambda)$ при порядке $\rho$ равномерно ограничена по $z \in E$. Положим

$$
B(\lambda ; f, E) \stackrel{\text { def }}{=} \sup _{z \in E} \ln |f(z, \lambda)|, \quad \lambda \in \mathbb{C}^{n},
$$

и точно так же, как в п. 1 , через функцию $B(\lambda ; f, E)$ определим взаимный индикатор $H_{\rho}^{*}(\lambda ; f, E)$ для семейства $f$ и множества $E$ при порядке $\rho$. При введенных ограничениях взаимньй индикатор - плюрисубгармоническая функция конечного типа при порядке $\rho$, а его сужение на единичную сферу - субсферическая функция порядка $\rho$. 
ТЕОРема 3.3. Пусть во введенных обозначениях и условиях сужение взаимного индикатора семейства $f$ и множества $Е$ на единичную сферу в $\mathbb{C}^{n}$ не превышает некоторой непрерывной субсферической функиии $h$ порядка $\rho, \Lambda \subset \mathbb{C}^{n}$. Если для $k \in \mathrm{SS}_{\gamma}^{+}$выполнено одно из соотношений 1)-3) теоремы 3.1, то система голоморфных функиий $\{f(z, \lambda): \lambda \in \Lambda\}$ полна на $E \subset \mathbb{C}^{p}$.

Доказательство этой теоремы практически не отличается от доказательства теоремы 3.2.

ЗАмечАниЕ. В случае, когда $\rho=1, f(z)=\exp \left(z_{1}+z_{2}+\cdots+z_{n}\right), z \in \mathbb{C}^{n}$, а $E$ - выпуклая область Рунге в $\mathbb{C}^{n}$, можно показать, что теорема 3.2 останется верной, если строгие неравенства ">" в соотношениях 1)-3) заменить на нестрогие " $\geqslant$ ". В такой ситуации теорема 3.2 точна. В этом можно убедиться, выбирая в качестве множества $\Lambda$ нулевые множества целых функций порядка 1 и вполне регулярного роста с радиальным индикатором сколь угодно близким снизу к опорной функции области $E$.

\section{СПИСОК ЦИТИРОВАННОЙ ЛИТЕРАТУРЫ}

[1] Гельфонд А. О. Sur les systèmes complets des fonctions analytiques // Матем. сб. 1938. T. 4(46). C. $149-156$.

[2] Маркушевич А. И. О базисе в пространстве аналитических функций // Матем. сб. 1945. T. $17(59)$. C. $211-252$.

[3] Ибрагимов И.И. О полноте системы аналитических функций $\left\{f\left(\alpha_{k} z\right)\right\} / /$ Изв. АН СССР. Сер. матем. 1949. Т. 13. № 1. С. 45-54.

[4] Ибрагимов И.И. Методы интерполяции функций и некоторые их применения. М.: Наука, 1971.

[5] Левин Б. Я. Распределение корней целых функций. М.: Физматгиз, 1956.

[6] Леонтьев А.Ф. О полноте одной системы аналитических функций // Матем. сб. 1952. T. 31(72). C. 381-413.

[7] Леонтьев А. Ф. Обобщения рядов экспонент. М.: Наука, 1981.

[8] Лохин И.Ф. О полноте систем функций вида $\left\{f\left(\lambda_{n} z\right)\right\} / /$ Докл. АН СССР. 1951. Т. 81. C. $141-155$.

[9] Лохин И.Ф. О полноте систем функций вида $\left\{f\left(\lambda_{n} z\right)\right\} / /$ Матем. сб. 1954. Т. 35(77). C. $215-222$.

[10] Хабибуллин Б. Н. Теорема единственности для субгармонических функций конечного порядка // Матем. сб. 1991. Т. 35. №6. С. 811-827.

[11] Гришин А.Ф., Содин М. Л. Рост по лучу, распределение корней по аргументам целой функции конечного порядка и одна теорема единственности // Теория функций, функциональный анализ и их приложения. Вып. 50. Харьков: Вища школа, 1988. С. 47-61.

[12] Хабибуллин Б. Н. Множества единственности в пространствах целых функций одной переменной // Изв. АН СССР. Сер. матем. 1991. Т. 55. № 5. С. 1101-1123.

[13] Ронкин Л.И. Целые функции // Итоги науки и техн. Соврем. пробл. матем. Фундамент. направления. Т. 9. М.: ВИНИТИ, 1986. С. 5-36.

[14] Груман Л., Лелон П. Целые функции многих комплексных переменных. М.: Мир, 1989.

[15] Ронкин Л.И. Введение в теорию целых функций многих переменных. М.: Наука, 1971.

[16] Кондратюк А. А. Сферические гармоники и субгармонические функции // Матем. сб. 1984. T. 125. № 2. C. 147-166.

[17] Чирка Е. М. Комплексные аналитические множества. М.: Наука, 1985.

[18] Хабибуллин Б. Н. Оценки объема нулевых множеств голоморфных функций // Изв. вузов. Матем. 1992. № 3. С. 58-63.

[19] Хейман У., Кеннеди П. Субгармонические функции. М.: Мир, 1980. 